\title{
$\mathbb{N}<\mathrm{T}$
}

trum

\section{On the Quality of Wall Time Estimates for Resource Allocation Prediction}

Mehmet Soysal, Marco Berghoff, Dalibor Klucasek and Achim Streit

Steinbuch Centre for Computing (SCC) / Scientific Computing and Simulation (SCS)

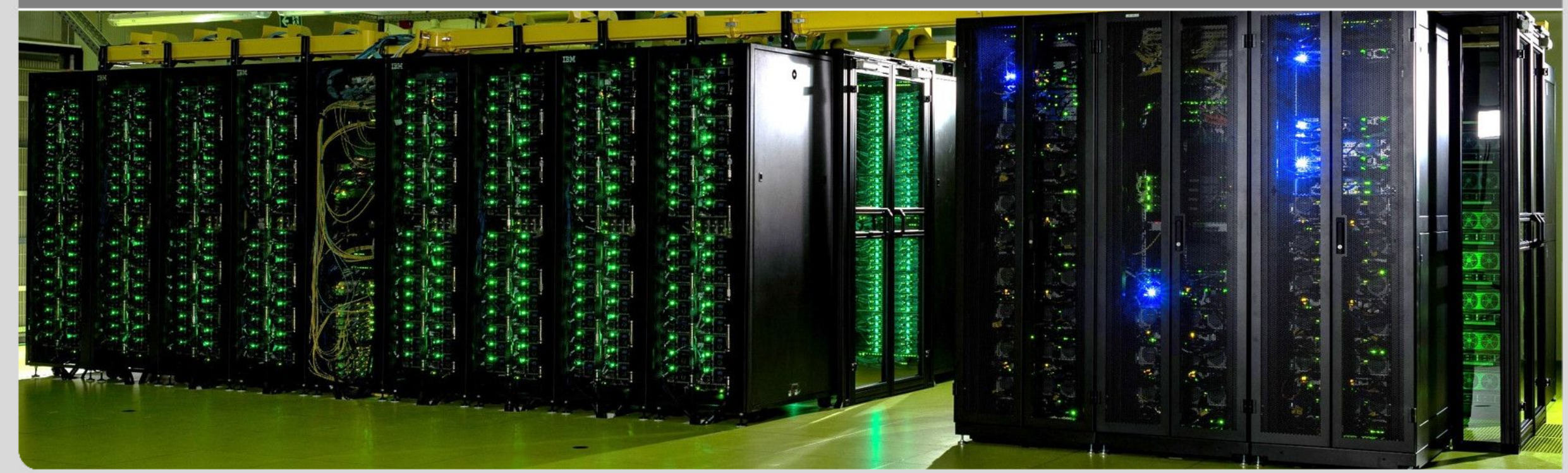




\section{Overview}

- Motivation

- Problem

- Metric

- Results

- Conclusion \& take away 


\section{Motivation}

- The I/O Subsystem (parallel FS) is a bottleneck in HPC Systems

- Bandwidth, metadata or latency

- Data Staging in advance to compute node

- Which nodes are going to be allocated?

- Wall times are far away from optimal

- How good wall time predictions have to be? 


\section{Goal: Data staging in advance}
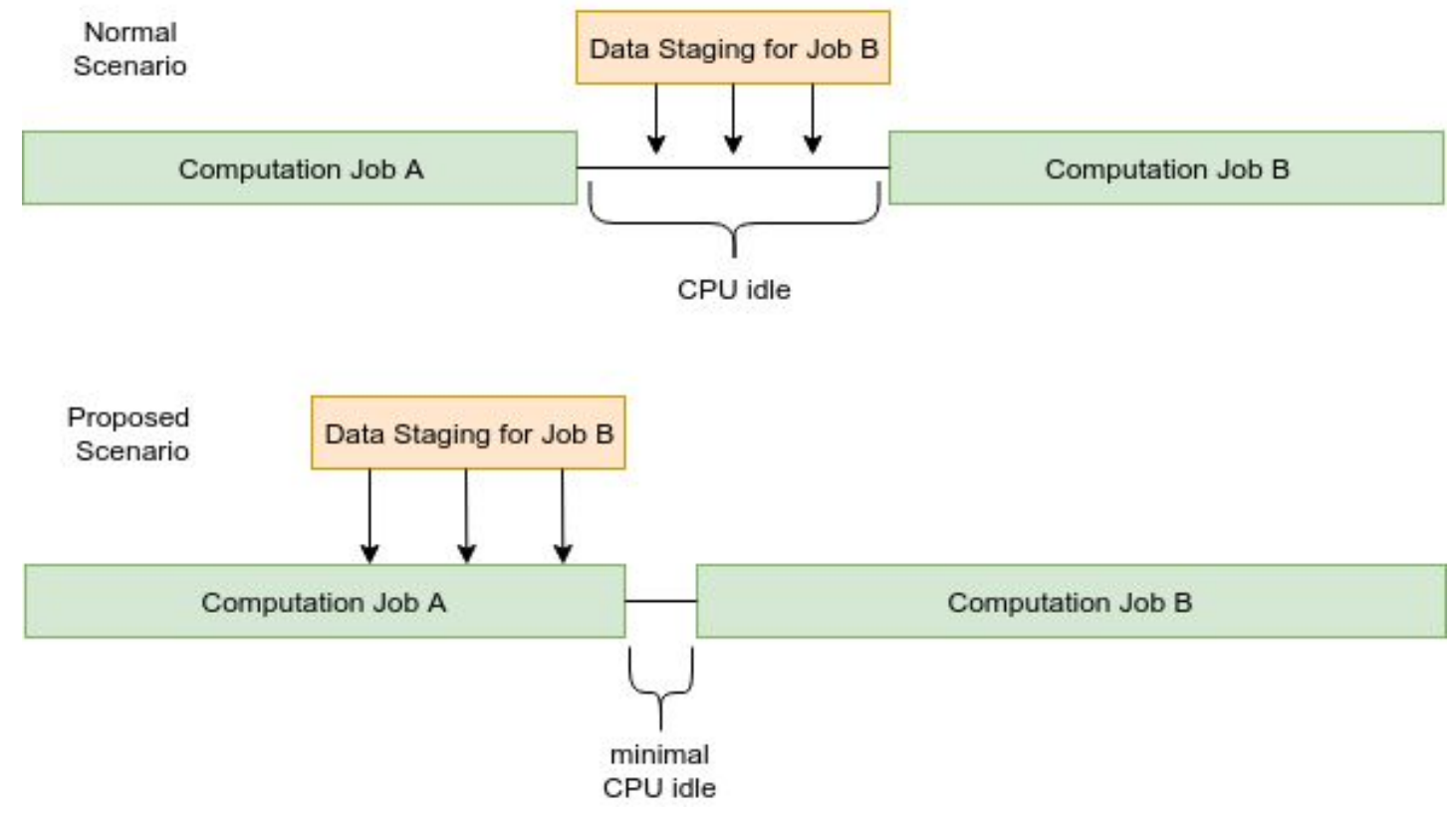

- Based on the allocation prediction

- No modification on scheduling behaviour

- How reliable is the schedule? 


\section{Scenario 1: Backfill-Scheduling}

Existing Schedule

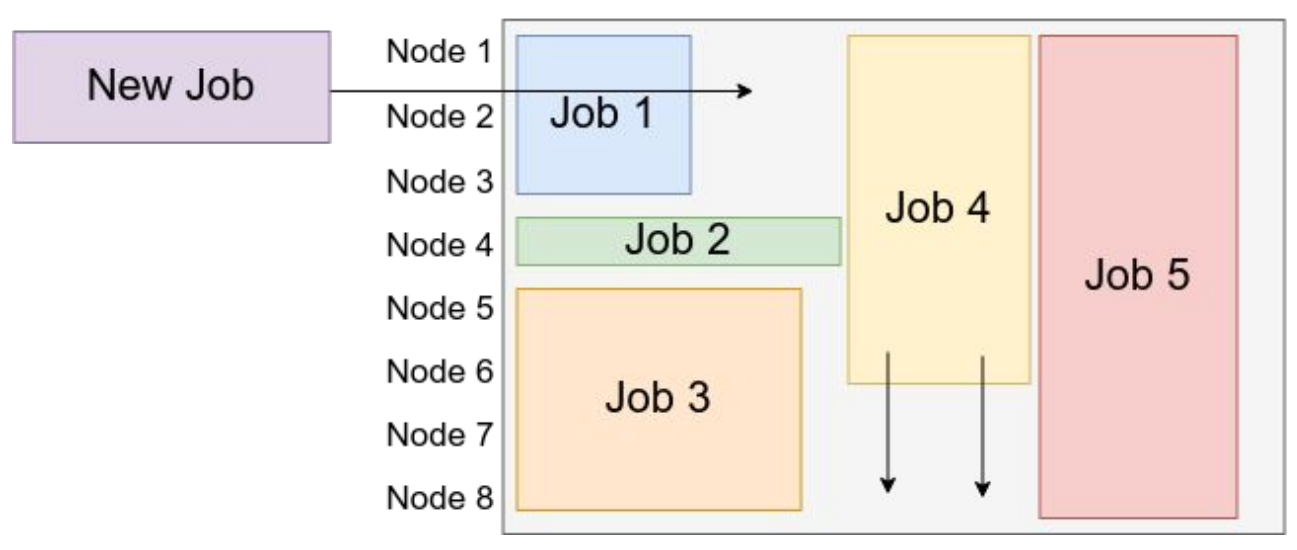

New Schedule

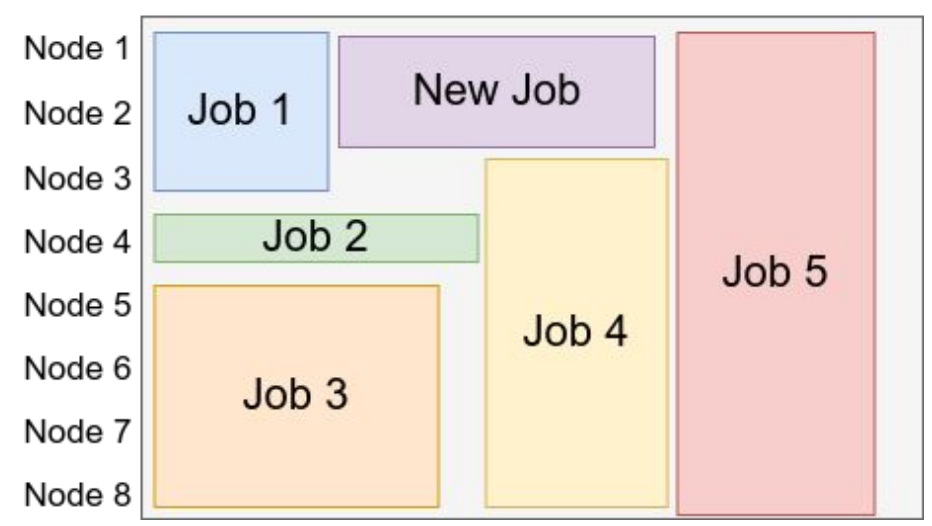

- Backfilling re-shuffles planned schedule 


\section{Scenario 2: Forward jump in schedule}

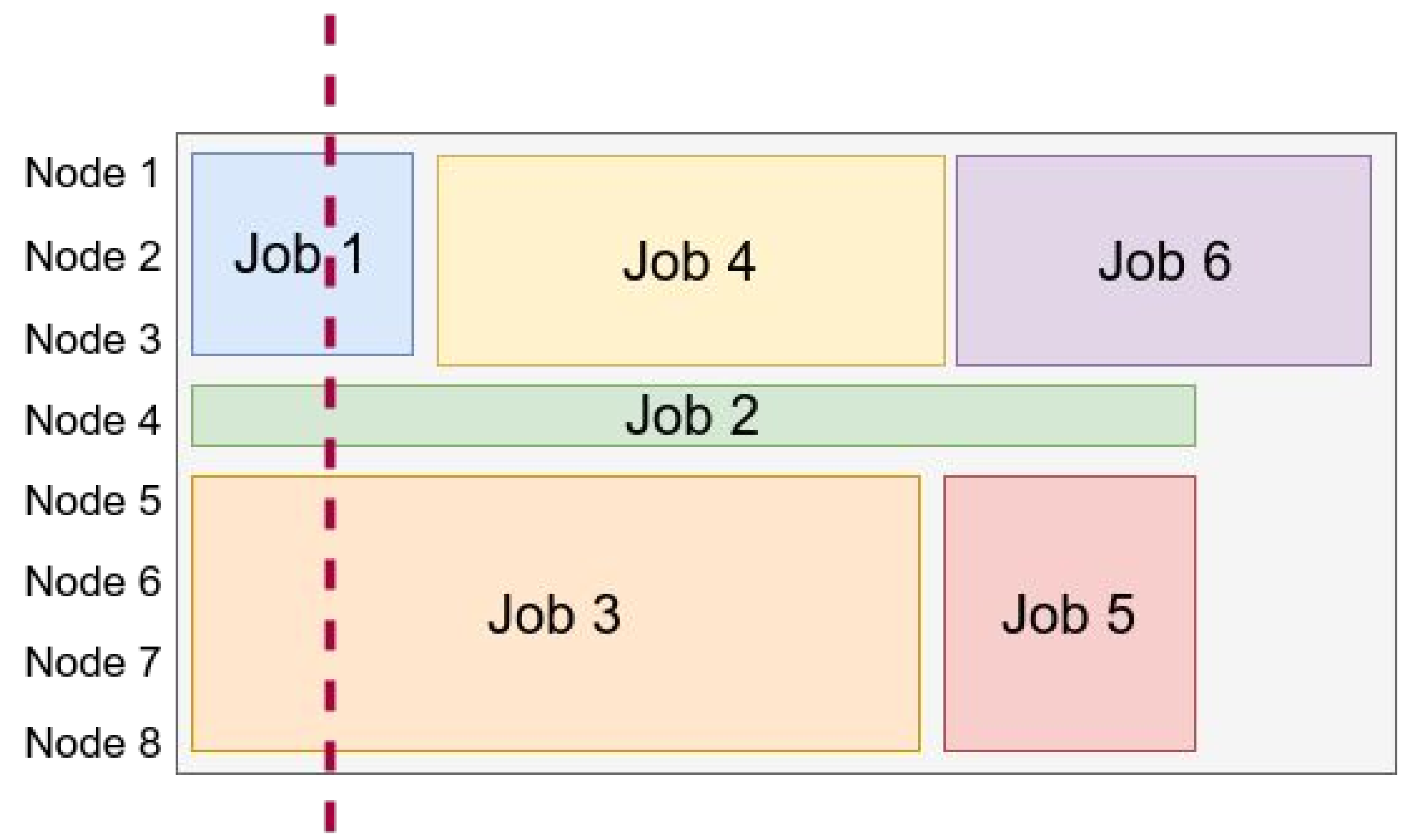




\section{Jump forward (2)}

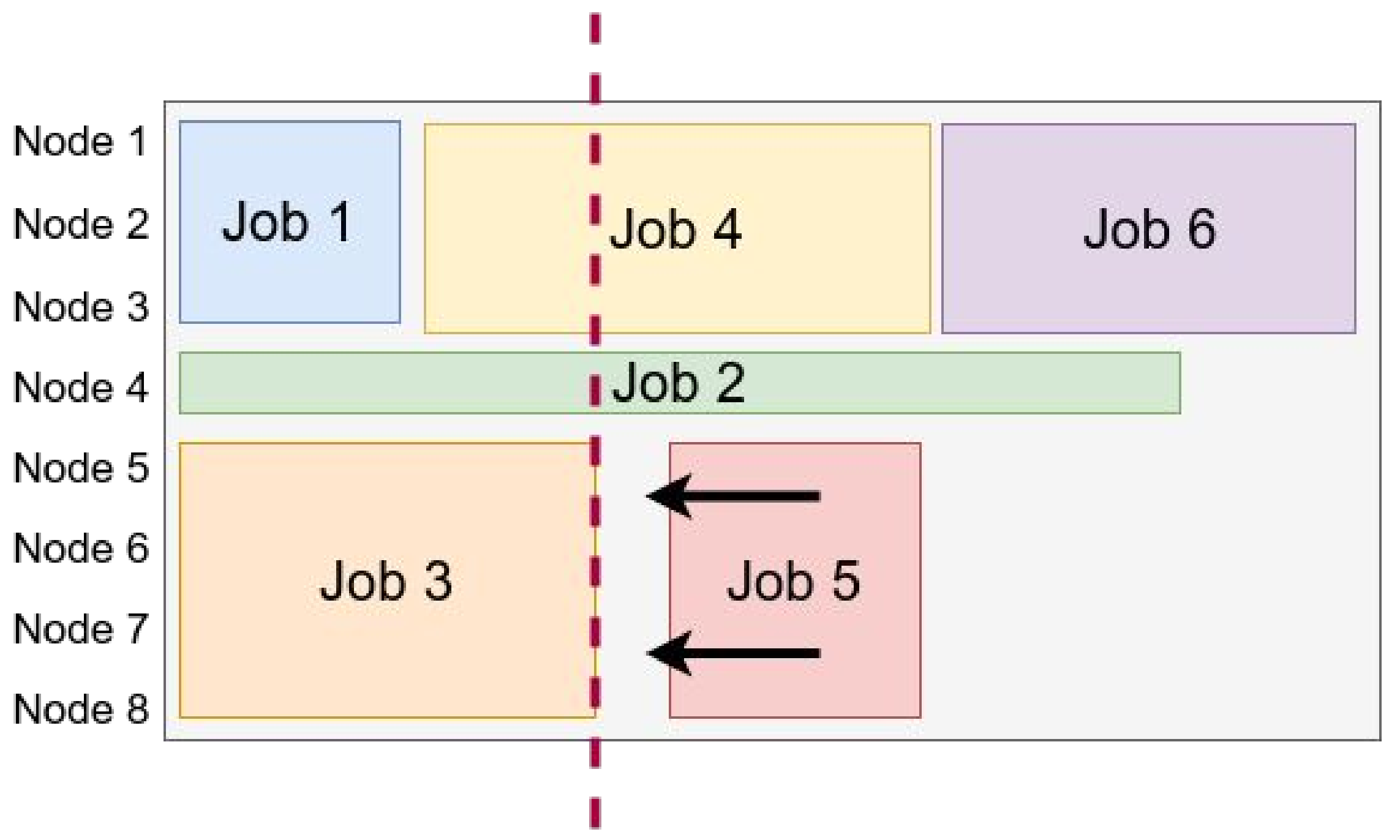




\section{How to solve}

- Many other cases cause reschedule

- Node failure

- Other nodes earlier free

- High priority jobs

- Need accurate wall time estimates

- Reduces need for back-filling

- No jumping forward in schedule

- Keep cluster utilization high $\leftarrow$

- Many approaches to predict wall time estimates Simple Rules, Machine Learning (ML), Automatic ML, Deep learning 


\section{Evaluation}

- Not another wall time predictor

- Impact of accurate wall time on node prediction

- Improve wall times artificially ("redefined" requested wall time)

- No under-estimations

- Workloads from the parallel workload archiv

- CTC, SDSC, KTH, ForHLR II*

- ALEA new feature developed

- Node allocation tracking 


\section{Redefined requested wall time (1)}

$\widetilde{T}_{R E Q}=T_{R E Q}+\lambda_{x}\left(T_{R E Q}-T_{U S E D}\right)$, for $x \in[0,1]$

Requested Wall time

\section{Batch Job}

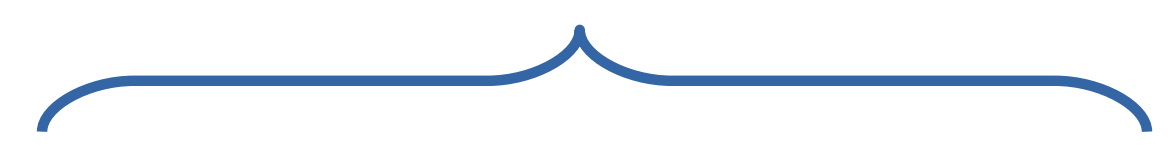

Used wall time

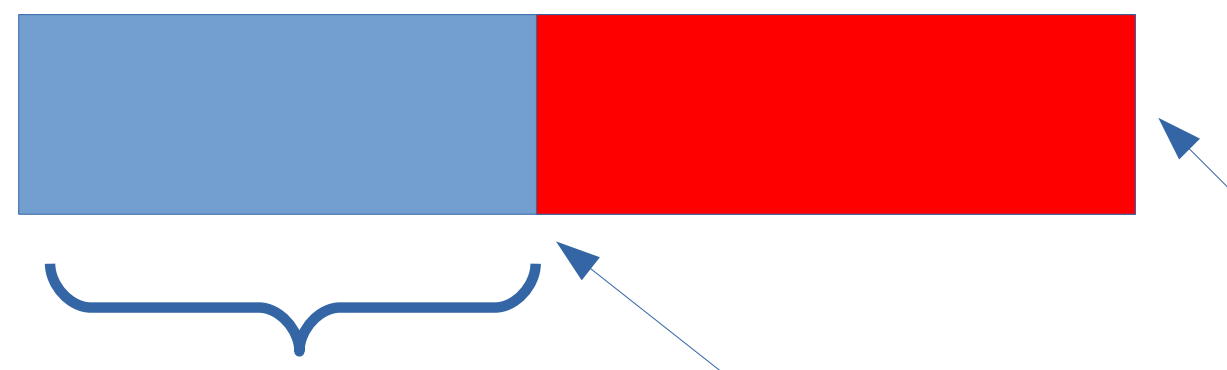

$$
\widetilde{T}_{R E Q}=\lambda_{0}
$$




\section{Redefined requested wall time (1)}

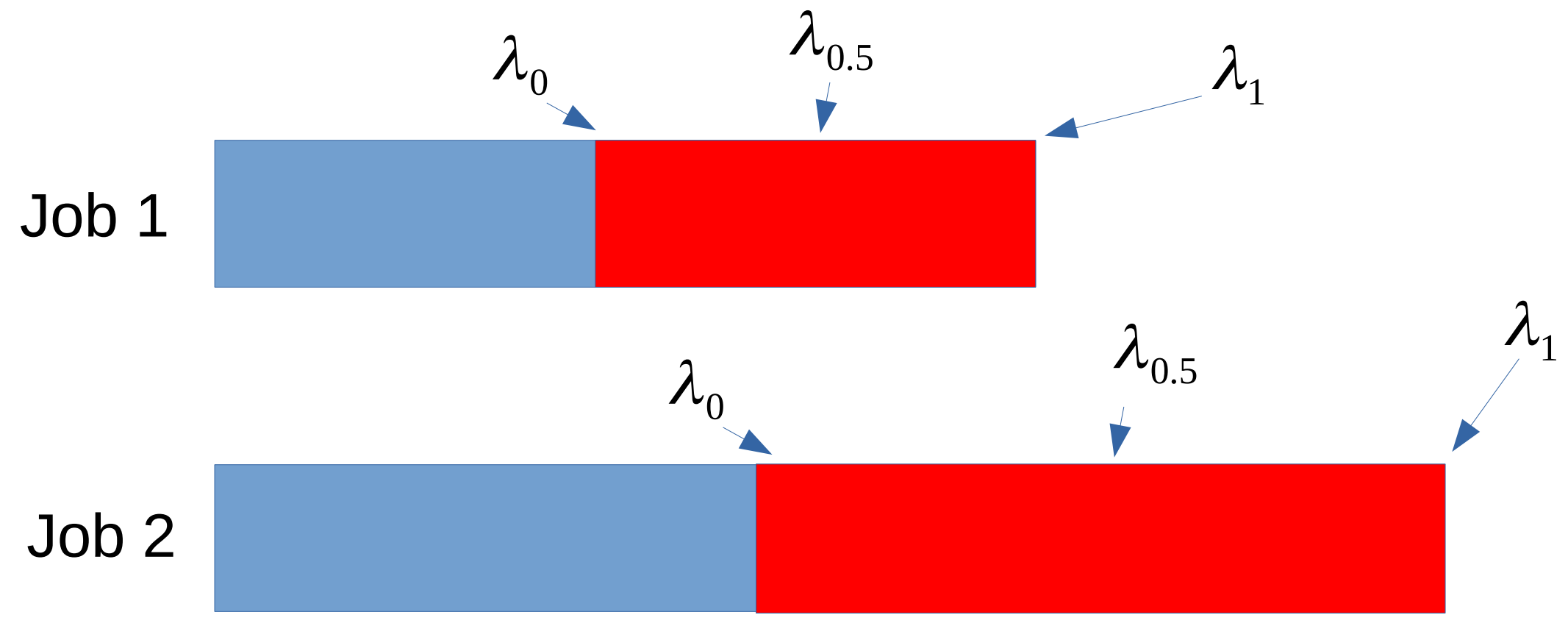

- Calculate for every job a new wall time estimate based on $\lambda_{x}$ for given $x$ 


\section{Requested / used walltime}

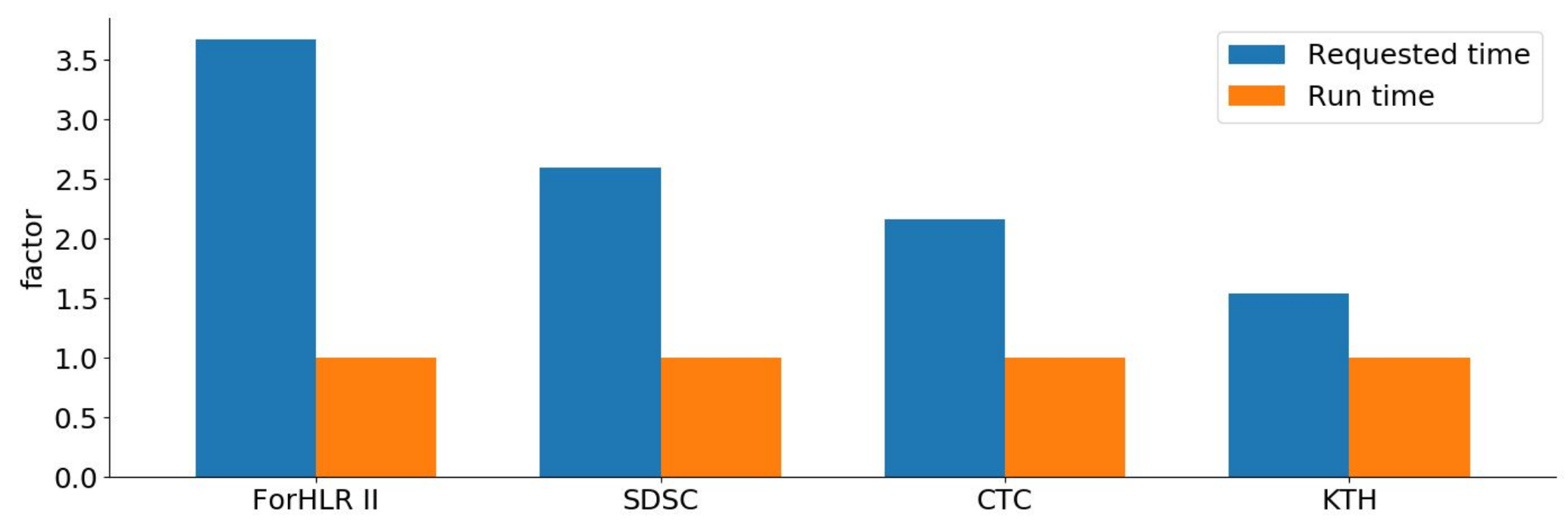

- User request more than they use

- ForHLR $3.5 \mathrm{X}$ requested wall time than used wall time SDSC 2.5X CTC 2.0X $\mathrm{KTH} \quad 1.5 \mathrm{X}$ 


\section{Improved wall times estimates}

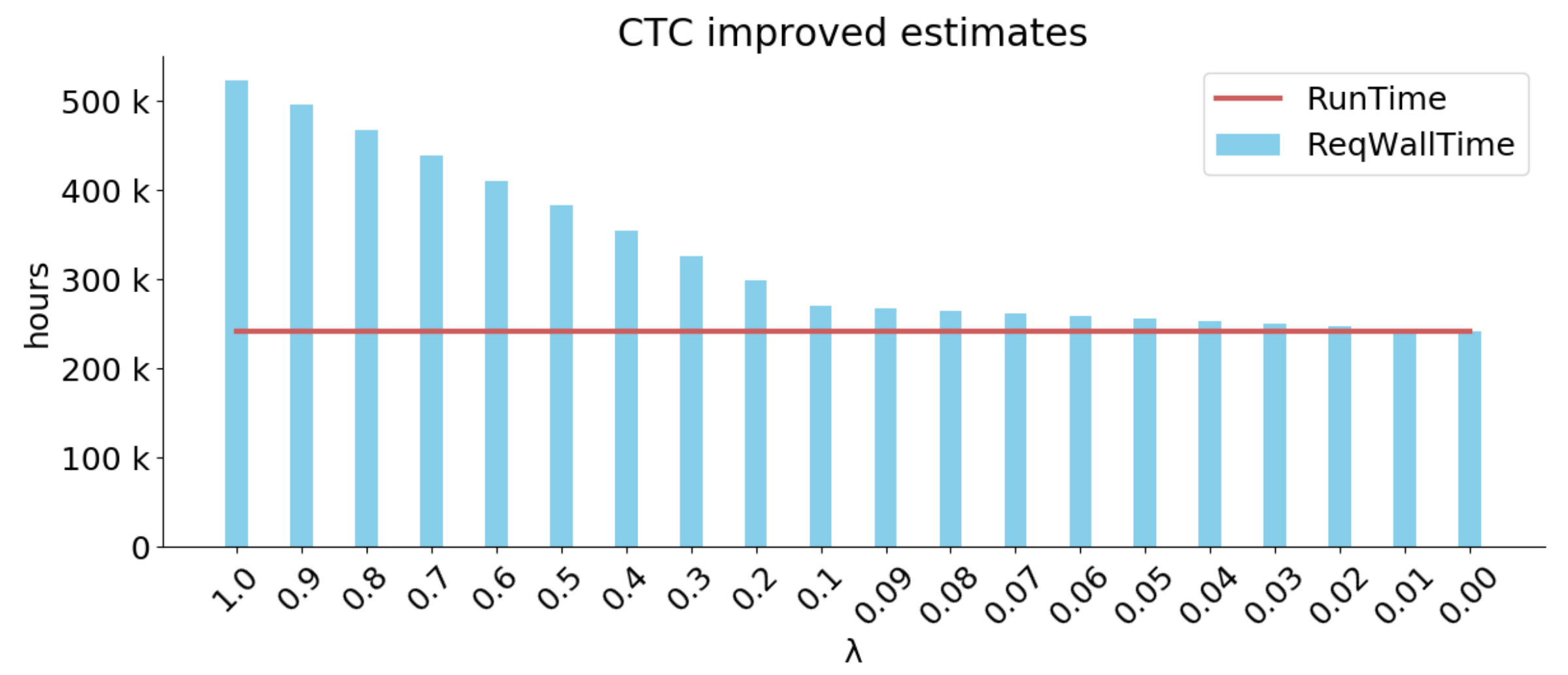




\section{Metric}

- Categorized into valid node allocation prediction time $\left(T_{\text {NAP }}\right)$

$>10$ minutes before job start the node allocation list is known

1 seconds -10 minutes

0 seconds -1 second

Instant started jobs 


\section{Results - CTC - FCFS}

CTC - FCFS - node_valid_for

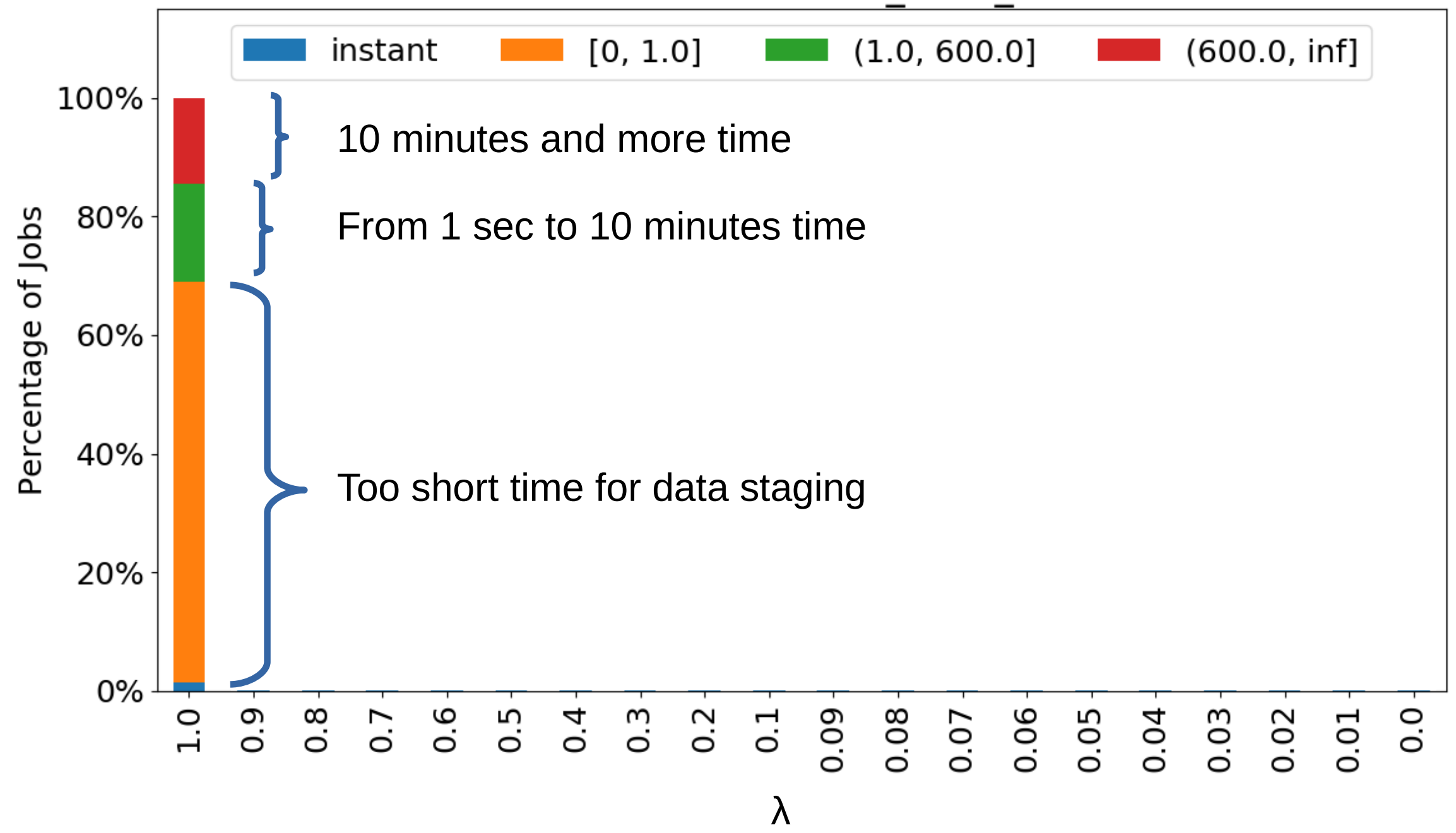




\section{Results - CTC - FCFS}

CTC - FCFS - node_valid_for

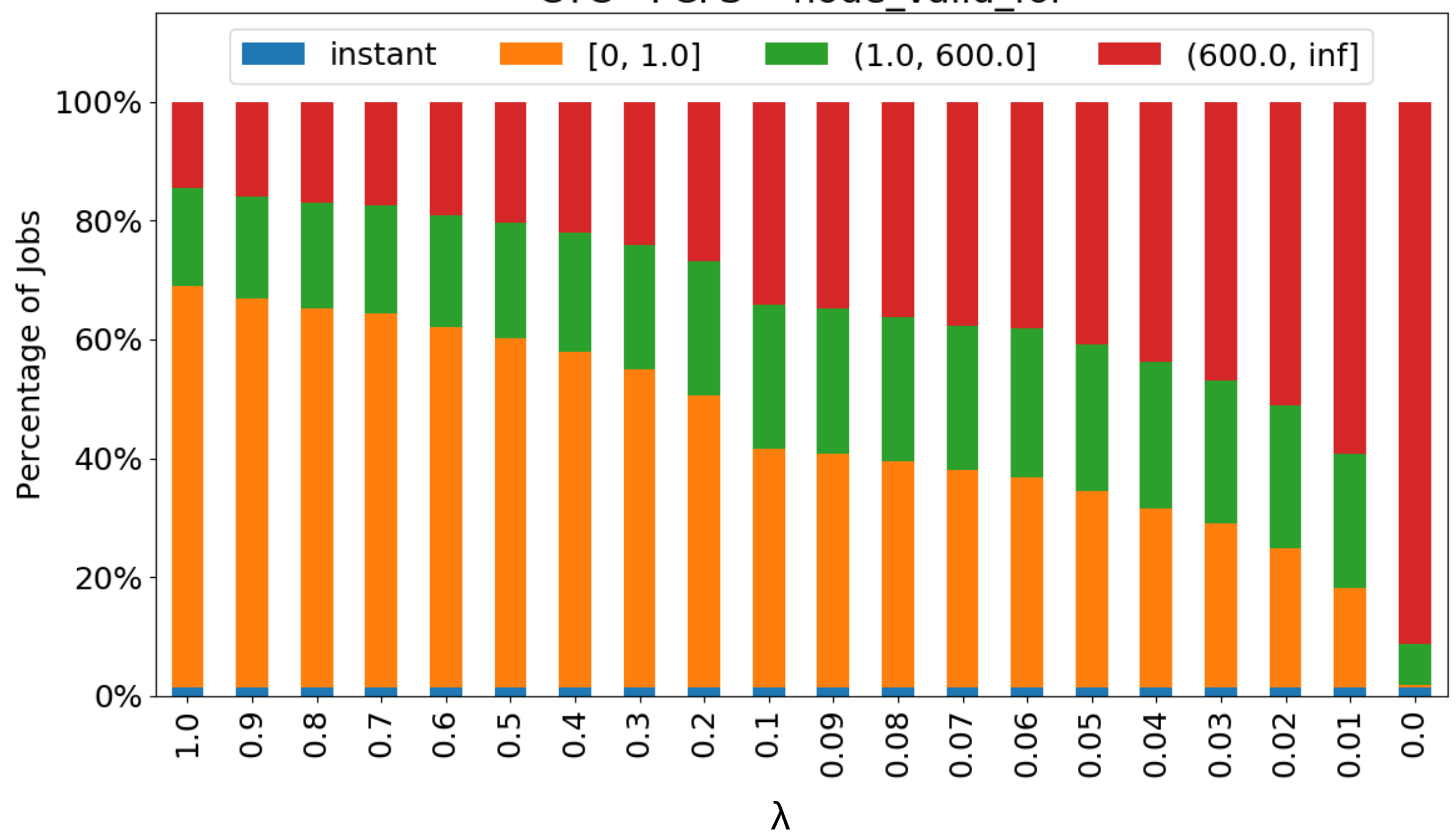




\section{Results - CTC - Back-filling}

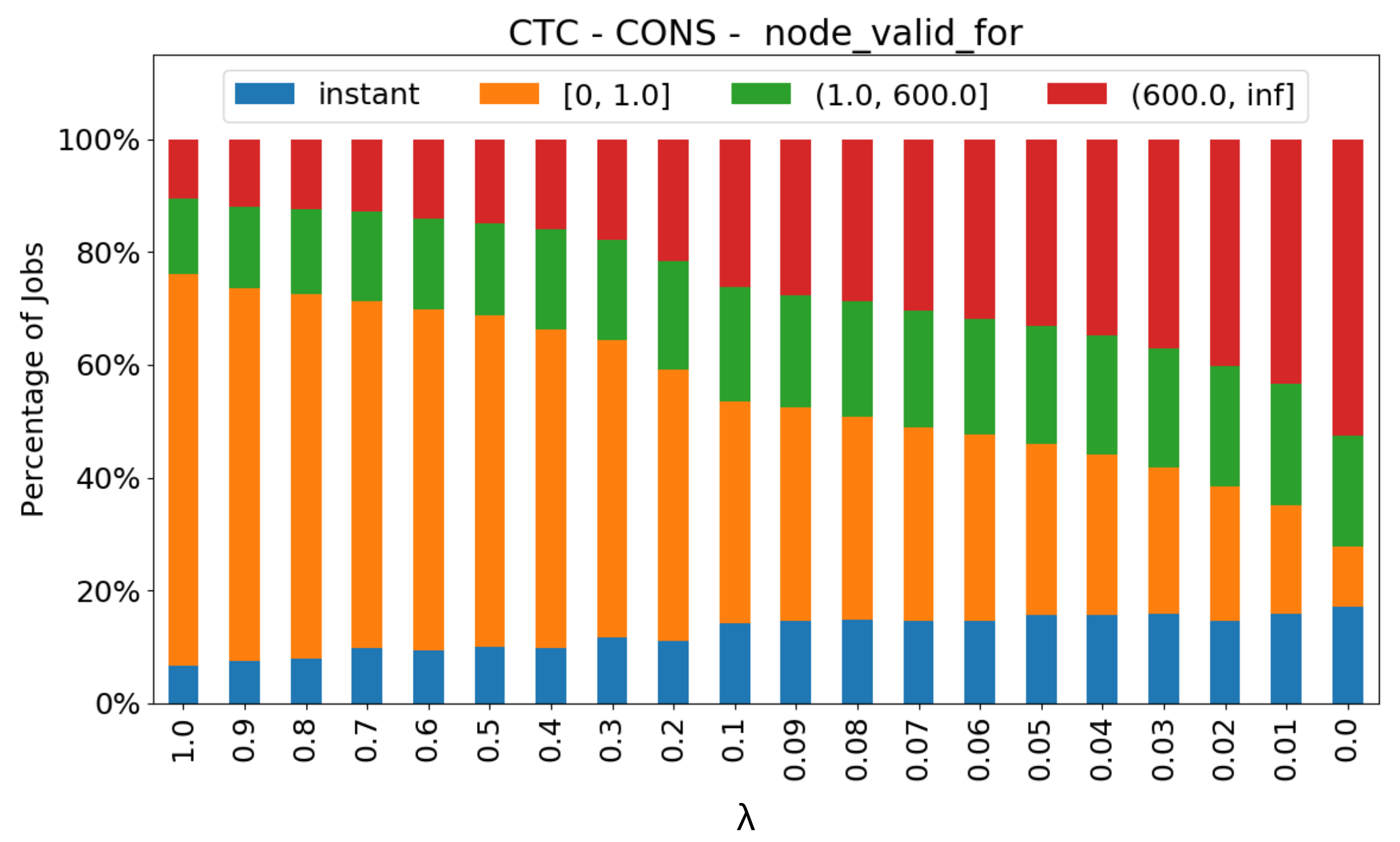




\section{Conclusion \& Take Away}

- With FCFS higher accuracy on node allocations

- Alea wall time predictor is quite good if user estimations are bad

- But still: Even with perfect wall times there is a huge uncertainty

- Alea can now simulate node allocation prediction

- Modification to scheduling needed for advanced data staging

- Reservations

- Slurm ODFS Burst buffer plugin 


\section{Acknowledgement}

- ADA-FS Project

- DFG priority programm SPPEXA "Software for exascale Computing"

- Steinbuch Centre for Computing

- Contact: Mehmet.Soysal@kit.edu

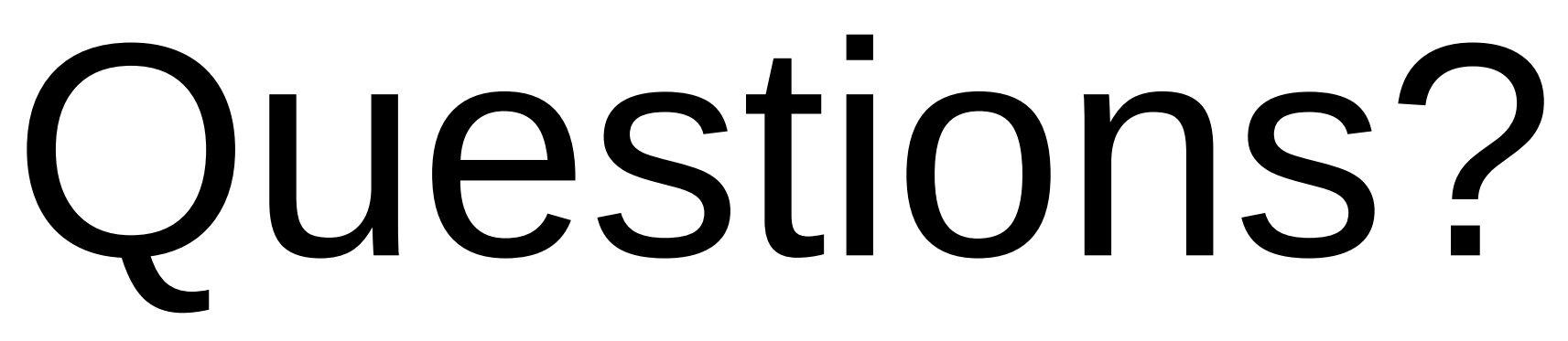

